\title{
Extensional flow dynamics of polystyrene melt
}

\author{
Huang, Qian; Rasmussen, H. K.
}

Published in:

Journal of Rheology

Link to article, DOI:

10.1122/1.5110027

Publication date:

2019

Document Version

Peer reviewed version

Link back to DTU Orbit

Citation (APA):

Huang, Q., \& Rasmussen, H. K. (2019). Extensional flow dynamics of polystyrene melt. Journal of Rheology, 63(5), 829-835. https://doi.org/10.1122/1.5110027

\section{General rights}

Copyright and moral rights for the publications made accessible in the public portal are retained by the authors and/or other copyright owners and it is a condition of accessing publications that users recognise and abide by the legal requirements associated with these rights.

- Users may download and print one copy of any publication from the public portal for the purpose of private study or research.

- You may not further distribute the material or use it for any profit-making activity or commercial gain

- You may freely distribute the URL identifying the publication in the public portal

If you believe that this document breaches copyright please contact us providing details, and we will remove access to the work immediately and investigate your claim 


\title{
Extensional flow dynamics of polystyrene melt
}

\author{
Qian Huang ${ }^{1}$ and Henrik Koblitz Rasmussen ${ }^{2, *}$ \\ (1) Department of Chemical and Biochemical Engineering, \\ (2) Department of Mechanical Engineering \\ Technical University of Denmark, DK-2800 Kgs. Lyngby, Denmark
}

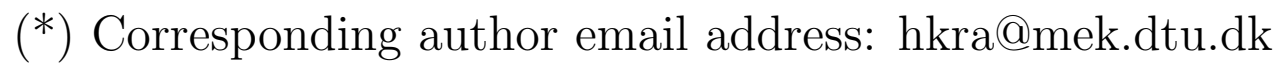

\begin{abstract}
A linear polystyrene melt with broad molecular weight distribution (CAS 0993-53-6 from Aldrich) was characterized in extensional flow using a filament stretching rheometer. A series of measurements were performed, including: 1) The startup of uniaxial extensional flow until steady state; 2) The strain controlled reversed (i.e. biaxial) flow following the startup of uniaxial flow; and 3) Stress relaxation (i.e. keeping the strain unchanged) also following the startup of uniaxial flow. The measurements were compared with the model by E. Narimissa and M. H. Wagner (J. Rheol. 60, 625-636, 2016). Good agreements at low extension rates and at low strain values were observed. Higher deviations were observed at high rates under steady state conditions as well as during stress relaxation and reversed flow.
\end{abstract}

\section{Introduction}

Understanding the flow behaviour of polymer melts is important for polymer processing operations. It is particularly the flow dynamics of polymer melts with broad molecular weight distribution, which is of interest. This is of course linked to the physics of theoretically more ideal systems such as mono and bidisperse melts. Constitutive models for broadly distributed polymer melts were initiated with phenomenological models such as the Giesekus [1, 2], Leonov [3] and Phan-Thien Tanner [4] models. These are versatile models, but commonly require a comprehensive experimental characterization to be used in the modelling of polymer melt flow. The most recent models were focused on the ability to predict the flow behaviour of ideal monodisperse polymer systems $[5,6,7,8,9,10]$. A large effort has been involved in developing the understanding from monodisperse to polydisperse melt systems $[6,11,12,13,14,15]$. However, theoretical ideas are always challenged by experiments. For example, even ideally monodisperse polymer melts have not shown the expected unique flow dependency with the number of entanglements $[16,17,18,19]$ which has been the common assumption.

Experimentally well characterized extensional flow is of particular importance in the validation of constitutive models. For broadly distributed polymer melts, the shear viscosity, compared to the linear viscoelastic properties, is not sensitive to the changes in the material's property [20]. By contrast, extensional flow is very sensitive to the changes in molecular weight distribution [14, 21], polymer structure [22] and composition [23]. A classic example is the importance of reversed extensional flow. Wagner and Stephenson [24] demonstrated that the assumption of the time and strain separability, a well-established principle in the linear viscoelastic regime, cannot be extended to the nonlinear region. The principle seemed an appropriate assumption in the startup of extensional flow [25], while a comparison with the strain recovery [24] showed a failure. The strain recovery is 
the maximal retraction of an initially stretched sample in uniaxial flow retracting without external stress. More recently Nielsen and Rasmussen [26] measured the reversed extensional flow under controlled strain conditions on a monodisperse polystyrene melt. They verified that the general constitutive equation suggested by Doi and Edwards for linear polymers $[27,28]$ is unable to capture the flow dynamics of polymer melts. The above examples show that the characterization of melt behaviour in the startup of extensional flow is important but not sufficient. The characterization under more complex extension conditions is critical. Previously narrow molar mass distributed (NMMD) linear [26, 29, 30] and branched (pom-pom) polystyrenes [31] have been characterized not only in startup of extensional flow, but also in stress relaxation and reversed flow. Here we present similar measurements on a broadly distributed linear polystyrene melt which has previously been characterized in its molecular weight distribution [32].

\section{Material and characterization}

The polystyrene used in this work is CAS 0993-53-6 from Aldrich with a glass transition temperature $\left(T_{g}\right)$ of $99^{\circ} \mathrm{C}$. The thermal properties and molecular weight distribution of this polystyrene have been characterized by Rasmussen and Eriksson [32]. The molecular weight distribution is shown in their figure 4 where the weight average molecular weight is $M_{w}=230 \mathrm{~kg} / \mathrm{mole}$ and the number average $M_{n}=60 \mathrm{~kg} /$ mole. The polystyrene contains a small amount of high molecular weight of above 1000 $\mathrm{kg} /$ mole. We are using the same CAS 0993-53-6 polystyrene batch as in Rasmussen and Eriksson [32]. Eriksson and Rasmussen [32] also characterized the polystyrene in mechanical spectroscopy using small amplitude oscillatory shear flow and their measurements are shown in Figure 1. These were identical within a few percent of our measurements. We have determined the linear viscoelastic memory function, $M(s)$, with a fitting to a sum of exponential function of

$$
M(s)=\sum_{i=1}^{N} \frac{g_{i}}{\tau_{i}} \exp \left(-s / \tau_{i}\right),
$$

where the moduli $g_{i}$ and relaxation times $\tau_{i}$ are listed in Table 1 . The corresponding calculated storage modulus, $G^{\prime}$, and loss modulus, $G^{\prime \prime}$, have been added in Figure 1.

The use of relaxation modes are of course a discretization and simplification of the relaxation time spectrum. Any nonlinear model prediction will depend on the particular discretization. To reduce the sensitivity a sufficient number of modes need to be applied.

\section{Measurements of extensional flow}

The measurements of extensional flow were carried out using a filament stretching rheometer (FSR) constructed in-house [33, 34]. Basically, it involves an extension of cylindrical shaped sample with initial diameter, $D_{\mathrm{i}}$ (all of $9 \mathrm{~mm}$ ), and initial length, $L_{i}$ (of $2.0 \mathrm{~mm}$ to $2.5 \mathrm{~mm}$ ). The mid-filament diameter $D(t)$ and extensional force $F$ are measured during extension. The extensional stress $\left(\sigma_{\mathrm{zz}}-\sigma_{\mathrm{rr}}\right)$ are calculated using the measured extensional force, $F$, divided by the mid-filament cross sectional area, as [35]

$$
\sigma_{z z}-\sigma_{r r}=\frac{F(t)-m_{f} g / 2}{\pi D(t)^{2} / 4} \cdot \frac{1}{1+\left(D(t) / D_{\mathrm{i}}\right)^{10 / 3} \cdot \exp \left(-A_{\mathrm{i}}^{3}\right) /\left(3 A_{\mathrm{i}}^{2}\right)},
$$

where $m_{f}$ is the mass of the sample, $g$ is the standard gravity [36] and $A_{\mathrm{i}}=2 L_{\mathrm{i}} / D_{\mathrm{i}}$ is the initial aspect ratio. $\sigma_{\mathrm{rr}}$ and $\sigma_{\mathrm{zz}}$ are respectively the radial and axial (in the extension direction) components 
of the stress tensor, $\boldsymbol{\sigma}$. Equation (2) involves the correction factor suggested by Rasmussen et al. [35]. It removes the shear contribution that may add to the measured force at small values of Hencky strain, pre-strain and aspect ratio, so that a correct initial extensional viscosity within $3 \%$ deviation can be obtained. In the case of a pre-stretch to an initial diameter of $D_{0}$, the correct use of Equation (2) requires that the sample has been pre-stretched at a rate considerably slower than the inverse of the largest relaxation time. The Hencky strain at the mid-filament plane is $\epsilon(t)=-2 \ln \left(D(t) / D_{0}\right)$ and the corresponding strain rate is $\dot{\epsilon}=d \epsilon / d t$. The initiation of the stretch is at the time $t=0$. Equation (2) theoretically calculates an average central extensional stress. It is commonly replaced with an assumption of stress uniformity in the mid-filament cross sectional area, as the local values stay at the same values within a few percent accuracy [37].

Three types of extensional measurements were performed: Startup of uniaxial extensional flow with constant strain rate until 1) the steady state [34]; 2) a strain of $\epsilon_{0}$, followed by stress relaxation; and 3) a strain of $\epsilon_{0}$, followed by reversed biaxial flow. During the startup, the constant strain rate is defined as $\dot{\epsilon}^{+}=\dot{\epsilon}$. In the biaxially reversed flow the constant rate is $\dot{\epsilon}^{-}=-\dot{\epsilon}$. In all the presented experiments the value of $|\dot{\epsilon}|$, i.e. $\dot{\epsilon}^{-}$and $\dot{\epsilon}^{+}$, is the same within the same experiment. A useful quantity to characterize the reversed flow behaviour is the particular strain where the extensional stress reaches zero (at time $t_{R}$ ). At larger reversed strain buckling may occur. The needed reversed strain or "recovery strain" $\left(\epsilon_{R}\right)$ to reach the zero extensional stress from the maximal imposed strain is defined as $\epsilon_{R}=\epsilon_{0}-\epsilon\left(t_{R}\right)$. For further details regarding the methodology and definitions please refer to Nielsen and Rasmussen [26]. To our knowledge, it is so far the only method to measure reversed flow with a controlled rate in extension, and has been applied for large amplitude oscillatory elongation flow as well [29]. During the stress relaxation, the mid filament strain is fixed at a value of $\epsilon_{0}$. It still requires a dynamic control of the sample as only the mid-filament diameter should be kept unchanged. The experimental details for stress relaxation can be found in Nielsen et al. [30].

The viscosities during startup flow of extension are given as $\bar{\eta}^{+}=\left(\sigma_{\mathrm{zz}}-\sigma_{\mathrm{rr}}\right) / \dot{\epsilon}^{+}$. In stress relaxation, where the strain rate is zero, the viscosities are defined from the preceding startup flow, thus given as $\bar{\eta}^{-}=\left(\sigma_{\mathrm{zz}}-\sigma_{\mathrm{rr}}\right) / \dot{\epsilon}^{+}$.

\section{Constitutive model}

The overall concept of chain stretch was suggested by Marrucci and co-workers [38, 39] and is not related to a particular model. It seems that so far only the molecular stress function (MSF) constitutive model $[40,41]$ has been demonstrated to be in agreement with the measured "recovery strain", for linear (nearly) monodisperse melts [26]. Though other constitutive frameworks are likely to show a similar agreement, some may not. Here we will discuss the extensional dynamics of the polystyrene melt based on the recent MSF model for polydisperse polymers suggested by Narimissa and Wagner [42]. The stress tensor in the model is given as

$$
\boldsymbol{\sigma}=\int_{-\infty}^{t}\left[\sum_{i=1}^{N} \frac{g_{i}}{\tau_{i}} \exp \left(\left(t-t^{\prime}\right) / \tau_{i}\right) f_{i}\left(\boldsymbol{x}, t, t^{\prime}\right)^{2}\right] 5\left\langle\frac{\left[\mathbf{E}\left(\boldsymbol{x}, t, t^{\prime}\right) \cdot \mathbf{u}\right]\left[\mathbf{E}\left(\boldsymbol{x}, t, t^{\prime}\right) \cdot \mathbf{u}\right]}{\left|\mathbf{E}\left(\boldsymbol{x}, t, t^{\prime}\right) \cdot \mathbf{u}\right|^{2}}\right\rangle d t^{\prime}
$$

where the initial value of the molecular stress functions is $f_{i}\left(\boldsymbol{x}, t^{\prime}, t^{\prime}\right)=1$. $\mathbf{E}\left(\boldsymbol{x}, t, t^{\prime}\right)$ is the displacement gradient tensor where its components are defined as $E_{\mathrm{jk}}\left(\boldsymbol{x}, t, t^{\prime}\right)=\partial x_{\mathrm{j}} / \partial x_{\mathrm{k}}{ }^{\prime}$, with $\mathrm{j}=1,2,3$ and $\mathrm{k}=1,2,3$. The coordinates $\boldsymbol{x}=\left(x_{1}^{\prime}, x_{2}^{\prime}, x_{3}^{\prime}\right)$ and $\boldsymbol{x}=\left(x_{1}, x_{2}, x_{3}\right)$ are the same particle in the past time, $t^{\prime}$, and present time, $t$, respectively. The coordinates are Cartesian. All angular brackets are unit sphere integrals, given as $\langle\ldots\rangle=1 /(4 \pi) \int_{|\mathbf{u}|=1} \ldots d \mathbf{u} . \mathbf{u}$ is the unit vector. Analytical solutions of all the unit sphere integrals can be found in Urakawa et al. (1995) [43]. In the following we have 
omitted the $\left(\boldsymbol{x}, t, t^{\prime}\right)$ in the notation for $f_{i}\left(\boldsymbol{x}, t, t^{\prime}\right)$. The molecular stress functions are

$$
\frac{\partial}{\partial t} f_{i}=f_{i} \frac{\partial}{\partial t}\langle\ln |\mathbf{E} \cdot \mathbf{u}|\rangle-3 \frac{f_{i}-1}{\tau_{i}}\left(1-\frac{2}{3} w_{i}^{2}\right)-\frac{2}{3} \frac{f_{i}^{2}\left(f_{i}^{3}-1\right)}{\tau_{i}} w_{i}^{2}
$$

where

$$
w_{i}^{2}=\sum_{j=1}^{N} \frac{g_{i}}{G_{D}} \exp \left(-\tau_{i} / \tau_{j}\right)
$$

If the above value is larger than 1 then $w_{i}^{2}=1$. Further $G_{D}$ is the dilution modulus [42]. A comprehensive and detailed discussion of the constitutive equation used here can be found in Narimissa and Wagner (2016) [42]. The only needed information in the model is the fitted linear viscoelastic moduli $g_{i}$ and relaxation times $\tau_{i}$, as listed in Table 1, and the dilution modulus [42], fitted to a value of $7000 \mathrm{~Pa}$ for a broadly distributed linear polystyrene [42] with $M_{w}=336 \mathrm{~kg} / \mathrm{mole}$ and $M_{n}=125$ $\mathrm{kg} /$ mole [42]. To our knowledge, the molecular weight distribution has not been reported for this particular polystyrene polymer. This model is strictly a model for linear polydisperse polymer melts. It has a similar functional framework as in the monodisperse model $[40,41]$ and is based on the same idea of the 'interchain pressure' [44] which controls the stretch of the polymer. In the model, the polydispersity is handled through a multimode approach by linking one nonlinear function to each linear relaxation time. Monodisperse polymers only use one nonlinear function controlled by the Rouse time. The multimode approach is classical. It originates from the first suggested differential constitutive equations $[2,3,4]$, which were initially introduced with the use of only one linear relaxation time, i.e. one exponential relaxation. The multimode approach is of course still not physically correct, but has served as an approximation and is currently still the most widely used method in flow modelling. It is not necessary to apply this multimode approximation in integral constitutive equations, but has been done so in Equation (3).

\section{Results and discussion}

Figure 2 shows the measured extensional viscosity, $\bar{\eta}^{+}=\left(\sigma_{\mathrm{zz}}-\sigma_{\mathrm{rr}}\right) / \dot{\epsilon}^{+}$, where the polystyrene sample is extended with a constant extensional strain rate, $\dot{\epsilon}^{+}$. The startup of the extension is initiated at the time $t=0$ where the sample is stress free. Series of similar measurements on different linear polydisperse melts have been published previously $[45,46]$. This type of measurement can be obtained with a variety of extensional techniques; but with the FSR a clearer steady elongation plateau at high strain values can be attained, particularly at high strain rates. Most constitutive models for broadly distributed linear polymers have been validated using this type of measurement. We have inserted the prediction of the model from Narimissa and Wagner [42] in Figure 2 and it fits the data quite well at low extension rates, as expected. The model was originally verified using broadly distributed polystyrene, high density and linear low-density polyethylene melts.

The first challenge for a particular model is stress relaxation. With the defined initial condition of the sample, the stress during the state of constant strain gives quantitative information about the internal relaxation mechanism of the polymer. The undeformed mid-filament plane of the sample during the stress relaxation removes the strain dependency. It leaves only the (nonlinear) time dependency. Combined with the startup of flow, which contains both strain and time dependency, these two types of measurements would be considered as a minimum for an experimental validation for any constitutive models. The relaxation measurements have been added onto the startup data in Figure 3. During the stress relaxation, the mid filament strain is fixed at a value of $\epsilon_{0}$, keeping the mid-filament diameter unchanged. The viscosities during startup flow of extension are given as $\bar{\eta}^{+}=\left(\sigma_{\mathrm{zz}}-\sigma_{\mathrm{rr}}\right) / \dot{\epsilon}^{+}$, where the strain rate, $\dot{\epsilon}^{+}$, is the one from the preceding startup flow. The 
relaxation is initiated from a Hencky strain of $\epsilon_{0}=3$, where the stress is close to the steady stress. The MSF model from Narimissa and Wagner [42] gives a good agreement at low extension rates and large times. There seems to be a somewhat larger deviation on the initial measured stresses during the relaxation, particularly at high extensional rates. It seems to be a consequence of the lower level of the steady extensional viscosities. We have compared the predictions of a pure configurational stress model $[27,28]$ on figure 4 (shown by the solid lines), corresponding to a lack of internal chain stretch (i.e. $f_{i}=1$ in Equation (3)), with the relaxation measurements, from figure 3. The use of $f_{i}=1$ in Equation (3) corresponds to a Doi and Edwards model [27, 28]. It has previously been shown clearly for monodisperse polymers that the pure configurational stress accurately controls the terminal relaxation stresses [47]. However, none of our measurements reach a pure configurational stress condition. This leaves the pure configurational stress assumption to be validated in broadly distributed polymer systems.

Reversed flow does not give a clear separation between time and strain dependency as the stress relaxation does; but as mentioned previously, it can show critical flaws in constitutive models. The change of flow direction gives information about the amount of work related to the structural, i.e. entropic, changes in the polymers.

A comparison between the measured "recovery strain" and the model by Narimissa and Wagner [42] has been shown in Figure 5. During the reversed (biaxial) flow, initiated at a strain of $\epsilon_{0}$, following (uniaxial) extension the constant rate is given as $\dot{\epsilon}^{-}=-\dot{\epsilon}^{+}$. Here $\dot{\epsilon}^{+}$is the constant extensional rate during the startup of flow. The "recovery strain" $\left(\epsilon_{R}\right)$ is the necessary reversed strain to reach zero extensional stress, i.e. $\epsilon_{R}=\epsilon_{0}-\epsilon\left(t_{R}\right)$. The circles represent the "recovery strain" with different extensional rates where the flow is reversed at a fixed value of Hencky strain of $\epsilon_{0}=3$. The bullets are the "recovery strain" with a fixed extensional rate but different imposed Hencky strain values. The imposed strain values are from just above the linear regime to extensional steady state. From a strain of about 3, the "recovery strain" values are the same within the experimental accuracy. The complete stress-strain measurements during the reversed extension as well as the startup of flow are shown in Figure 6 and 7. Before the steady stress is reached, the predictions of the model by Narimissa and Wagner [42] are accurate, while more discrepancy is observed at larger strain values.

In all the previous computations a dilution modulus of $7000 \mathrm{~Pa}$ was used in the model as in [42]. This shows a good agreement with the experimental data at low extension rates, low strain values and at large time scales. To illustrate the sensitivity of the model towards the value of the dilution modulus we have applied a dilution modulus of $35000 \mathrm{~Pa}$ in figure 8 . Overall the change of the dilution modulus does not overall improve the agreement between model and data.

\section{Summary and conclusion}

We have presented a series of measurements on the startup of extension, as well as stress relaxation and reversed flow both following the startup of extension, for a polystyrene melt with broad molecular weight distribution. Similar types of measurements have been published previously for a narrow molar mass distributed (145 kg/mole) polystyrene. According to our knowledge, such measurements have not been presented for a broadly distributed linear polymer melt before.

We have given a detailed fitting of the small amplitude oscillatory shear flow measurements on the broadly distributed polystyrene melt with (exponential) Maxwell modes, allowing direct comparison with differential constitutive models. Some of these models may be either approximations or simplifications of more complex constitutive models.

We have made a comparison between our measurements and the model by Narimissa and Wagner [42] which is based on the ideas from Wagner et al. [40,41]. The model shows a good agreement with the experimental data at low extension rates, low strain values and at large time scales, using the 
same value of the dilution modulus of $7000 \mathrm{~Pa}$ as in [42] for polystyrene. Higher deviation is observed at high rates, towards steady state conditions and measurements following steady state conditions. It may be linked to the use of a multimode approach. Narimissa and Wagner [42] used a classical multimode approach, having one exponential linear relaxation linked to each nonlinear stretch. It is empirical and cannot be expected to have an exact physics. Notice that the integral constitutive equation from Narimissa and Wagner [42] is fundamentally not different from phenomenologically based multimode differential constitutive equations. We have here used the dilution modulus as is given by Narimissa and Wagner (2016) [42]. It is a parameter fitted to a series of extensional data on a polystyrene. Changing the value of the dilution modulus does not give an overall improved agreement with our measurements.

With the previously published molecular weight distribution of the used polystyrene, a theoretically complete characterization of the melt is possible, allowing the use of the data to validate all previously published constitutive models for polymer melts. New ideas can now be tested more comprehensively.

\section{Acknowledgements}

This work is supported by the Independent research fund Denmark, grant number 8022-00042B. 


\section{References}

[1] Giesekus, H., "Die Elastizität von Flüssigkeiten," Rheologica Acta 5, 29-35 (1966).

[2] Giesekus, H., "A simple constitutive equation for polymer fluids based on the concept of deformation dependent tensorial mobility," Journal of Non-Newtonian Fluid Mechanics 11, 69-109 (1982).

[3] Leonov, A. I., "Nonequilibrium thermodynamics and rheology of viscoelastic polymer media," Rheologica Acta 15, 85-98 (1976).

[4] Phan-Thien, N., and R. I. Tanner, "A New Constitutive Equation Derived From Network Theory," Journal of Non-Newtonian Fluid Mechanics 2, 353-365 (1977).

[5] Wagner, M. H., "Scaling relations for elongational flow of polystyrene melts and concentrated solutions of polystyrene in oligomeric styrene," Rheologica Acta 53, 765-777 (2014).

[6] Yaoita, T., T. Isaki, Y. Masubuchi, H. Watanabe, G. Ianniruberto, and G. Marrucci, "Primitive Chain Network Simulation of Elongational Flows of Entangled Linear Chains: Stretch/Orientation-induced Reduction of Monomeric Friction," Macromolecules 45, 2773-2782 (2012).

[7] Ianniruberto, G., A. Brasiello, and G. Marrucci, "Simulations of fast shear flows of PS oligomers confirm monomeric friction reduction in fast elongational flows of monodisperse PS melts as indicated by rheooptical data," Macromolecules 45, 8058-8066 (2012).

[8] Masubuchi, Y., Y. Matsumiya, and H. Watanabe, "Test of Orientation/Stretch-Induced Reduction of Friction via Primitive Chain Network Simulations for Polystyrene, Polyisoprene, and Poly(n.butyl acrylate)," Macromolecules 47, 6768-6775 (2014).

[9] Ianniruberto, G., "Extensional flows of solutions of entangled polymers confirm reduction of friction coefficient," Macromolecules 48, 6306-6312 (2015).

[10] Park, G. W., and G. Ianniruberto, "Flow-Induced Nematic Interaction and Friction Reduction Successfully Describe PS Melt and Solution Data in Extension Startup and Relaxation," Macromolecules 50, 4787-4796 (2017).

[11] Andreev, M., R. N. Khaliullin, R. J. A. Steenbakkers, and J. D. Schieber, "Approximations of the discrete slip-link model and their effect on nonlinear rheology predictions," Journal of Rheology 57, 535-557 (2013).

[12] Park, J. , D. W. Mead, and M.M. Denn, "Stochastic simulation of entangled polymeric liquids in fast flows: Microstructure modification," Journal of Rheology 56, 1057-1081 (2012).

[13] Read, D. J. , D. Auhl, C. Das, J. den Doelder, M. Kapnistos, I. Vittorias, and T. C. B. McLeish, "Linking Models of Polymerization and Dynamics to Predict Branched Polymer Structure and Flow," Science 333, 1871-1874 (2011).

[14] Auhl D., P. Chambon, T. C. B. McLeish, and D. J. Read, "Elongational flow of blends of long and short polymers: effective stretch relaxation time," Physical Review Letters 103, 136001 (2009). 
[15] Dhole, S., A. Leygue, C. Bailly, and R. Keunings, "A single segment differential tube model with interchain tube pressure effect," Journal of Non-Newtonian Fluid Mechanics 161, 10-18 (2009).

[16] Bach, A., K. Almdal, H. K. Rasmussen, and O. Hassager, "Elongational viscosity of narrow molar mass distribution polystyrene," Macromolecules 36, 5174-5179 (2003).

[17] Sridhar, T., M. Acharya, D. A. Nguyen, and P. K. Bhattacharjee, "On the Extensional Rheology of Polymer Melts and Concentrated Solutions," Macromolecules 47, 379-386 (2014).

[18] Nielsen, J. K., O. Hassager, H. K. Rasmussen, and G. H. McKinley, "Observing the chain stretch transition in a highly entangled polyisoprene melt using transient extensional rheometry," Journal of Rheology 53, 1327-1346 (2009).

[19] Rasmussen, H. K., S. L. Wingstrand, and O. Hassager, "On the universality in the extensional rheology of monodisperse polymer melts and oligomer dilutions thereof," Rheologica Acta https://doi.org/10.1007/s00397-019-01156-w.

[20] Cox, W. P., and E. H. Merz, "Correlation of dynamic and steady flow viscosities," Journal of Polymer Science 28, 619-622 (1958).

[21] Nielsen, J. K., H. K. Rasmussen, O. Hassager, and G. H. McKinley, "Elongational viscosity of monodisperse and bidisperse polystyrene melts," Journal of Rheology 50, 453-476 (2006).

[22] Nielsen, J. K., H. K. Rasmussen, M. Denberg, K. Almdal, and O. Hassager, "Nonlinear branchpoint dynamics of multiarm polystyrene," Macromolecules 39, 8844-8853 (2006).

[23] Huang, Q., O. Mednova, H. K. Rasmussen, N. J. Alvarez, A. L. Skov, K. Almdal, and O. Hassager, "Concentrated Polymer Solutions are Different from Melts: Role of Entanglement Molecular Weight," Macromolecules 46, 5026-5035 (2013).

[24] M. H. Wagner, and S. E. Stephenson, "The Irreversibility Assumption of Network Disentanglement in Flowing Polymer Melts and its Effects on Elastic Recoil Predictions," Journal of Rheology 23, 489-504 (1979).

[25] Wagner, M. H., "A constitutive analysis of uniaxial elongational flow data of a low-density polyethylene melt," Journal of Non-Newtonian Fluid Mechanics 4, 39-55 (1978).

[26] Nielsen, J. K., and H. K. Rasmussen, "Reversed extension flow," Journal of Non-Newtonian Fluid Mechanics 155, 15-19 (2008).

[27] Doi, M., S.F. Edwards "Dynamics of concentrated polymer systems. III. Constitutive equation," Journal of the Chemical Society, Faraday Transactions II 74, 1818-1832 (1978).

[28] Doi, M., and S.F. Edwards, The Theory of Polymer Dynamics (Clarendon Press, Oxford, 1986).

[29] Rasmussen, H. K., P. Laillé, and K. Yu, "Large amplitude oscillatory elongation flow," Rheologica Acta 47, 97-103 (2008).

[30] Nielsen, J. K., H. K. Rasmussen, and O. Hassager, "Stress relaxation of narrow molar mass distribution polystyrene following uniaxial extension," Journal of Rheology 52(4) 885-899 (2008).

[31] Rasmussen, H. K., A. L. Skov, J. K. Nielsen, and P. Laillé, "Elongational dynamics of multiarm polystyrene," Journal of Rheology 53, 401-415 (2009). 
[32] Rasmussen, H. K., and T. G. Eriksson, "Gas displacement of polymer melts in a cylinder: Experiments and viscoelastic simulations," Journal of Non-Newtonian Fluid Mechanics 143, 1-9 (2007).

[33] Rasmussen, H. K., J. H. Christensen, and S. J. Gøttsche, "Inflation of polymer melts into elliptic and circular cylinders," Journal of Non-Newtonian Fluid Mechanics 93, 245-263 (2000).

[34] Marin, J. M. R., J. K. Huusom, N. J. Alvarez, Q. Huang, H. K. Rasmussen, A. Bach, A. L. Skov, and O. Hassager, "A control scheme for filament stretching rheometers with application to polymer melts," Journal of Non-Newtonian Fluid Mechanics 194, 14-22 (2013).

[35] H. K. Rasmussen, A. G. Bejenariu, O. Hassager, and D. Auhl, "Experimental evaluation of the pure configurational stress assumption in the flow dynamics of entangled polymer melts," Journal of Rheology 54, 1325-1336 (2010).

[36] Szabo, P., "Transient filament stretching rheometer part I: Force Balance analysis," Rheologica Acta 36, 277-284 (1997).

[37] Nielsen, J. K., H. K. Rasmussen, and O. Hassager, "Stress relaxation of narrow molar mass distribution polystyrene following uni-axial extension," Journal of Rheology 52, 885-899 (2008).

[38] Marrucci, G., and B. de Cindio, "The stress relaxation of molten PMMA at large deformations and its theoretical interpretation," Rheologica Acta 19, 68-75 (1980).

[39] Marrucci, G., and N. Grizzuti, "Fast flows of concentrated polymers - predictions of the tube model on chain stretching," Gazzetta Chimica Italiana 118, 179-185 (1988).

[40] Wagner, M. H., S. Kheirandish, and O. Hassager, "Quantitative prediction of transient and steady-state elongational viscosity of nearly monodisperse polystyrene melts," Journal of Rheology 49, 1317-1327 (2005).

[41] Wagner, M. H., and V. H. Rolón-Garrido, "The interchain pressure effect in shear rheology," Rheologica Acta 49, 459-471 (2010).

[42] Narimissa, E., and M. H. Wagner, "A hierarchical multimode molecular stress function model for linear polymer melts in extensional flows," Journal of Rheology 60, 625-636 (2016).

[43] Urakawa, O., M. Takahashi, T. Masuda, and N. G. Ebrahimi, "Damping functions and chain relaxation in uniaxial and biaxial extensions: Comparison with the Doi-Edwards theory," Macromolecules 28, 7196-7201 (1995).

[44] Marrucci, G., and G. Ianniruberto, "Interchain pressure effect in extensional flows of entangled polymer melts," Macromolecules 37, 3934-3942 (2004).

[45] Bastian, H., Non-linear viscoelasticity of linear and long-chainbranched polymer melts in shear and extensional flows (Ph.D. thesis, Universität Stuttgart, Stuttgart, Germany, 2001).

[46] Hachmann, P., Multiaxiale dehnung von polymerschmelzen (Ph.D. thesis, ETH Zurich, Zurich, Switzerland, 1996).

[47] Huang, Q., and H. K. Rasmussen, "Stress relaxation following uniaxial extension of polystyrene melt and oligomer dilutions," Journal of Rheology 60, 465-471 (2016). 
Table 1: Linear viscoelastic parameters of the (CAS 0993-53-6 from Aldrich) polystyrene at $120^{\circ} \mathrm{C}$.

\begin{tabular}{r|ll}
\hline$i$ & $\tau_{i}$ & $g_{i}$ \\
\hline 1 & $692346 \mathrm{~s}$ & $162 \mathrm{~Pa}$ \\
2 & $238334 \mathrm{~s}$ & $311 \mathrm{~Pa}$ \\
3 & $83827 \mathrm{~s}$ & $2245 \mathrm{~Pa}$ \\
4 & $23267 \mathrm{~s}$ & $6770 \mathrm{~Pa}$ \\
5 & $6561 \mathrm{~s}$ & $13784 \mathrm{~Pa}$ \\
6 & $2067 \mathrm{~s}$ & $16768 \mathrm{~Pa}$ \\
7 & $840.9 \mathrm{~s}$ & $17710 \mathrm{~Pa}$ \\
8 & $298.8 \mathrm{~s}$ & $26470 \mathrm{~Pa}$ \\
9 & $94.31 \mathrm{~s}$ & $30903 \mathrm{~Pa}$ \\
10 & $25.12 \mathrm{~s}$ & $45594 \mathrm{~Pa}$ \\
11 & $5.619 \mathrm{~s}$ & $72394 \mathrm{~Pa}$ \\
12 & $1.229 \mathrm{~s}$ & $141937 \mathrm{~Pa}$ \\
13 & $0.3145 \mathrm{~s}$ & $221756 \mathrm{~Pa}$ \\
14 & $0.09437 \mathrm{~s}$ & $562067 \mathrm{~Pa}$ \\
15 & $0.001297 \mathrm{~s}$ & $80223274 \mathrm{~Pa}$ \\
\hline
\end{tabular}




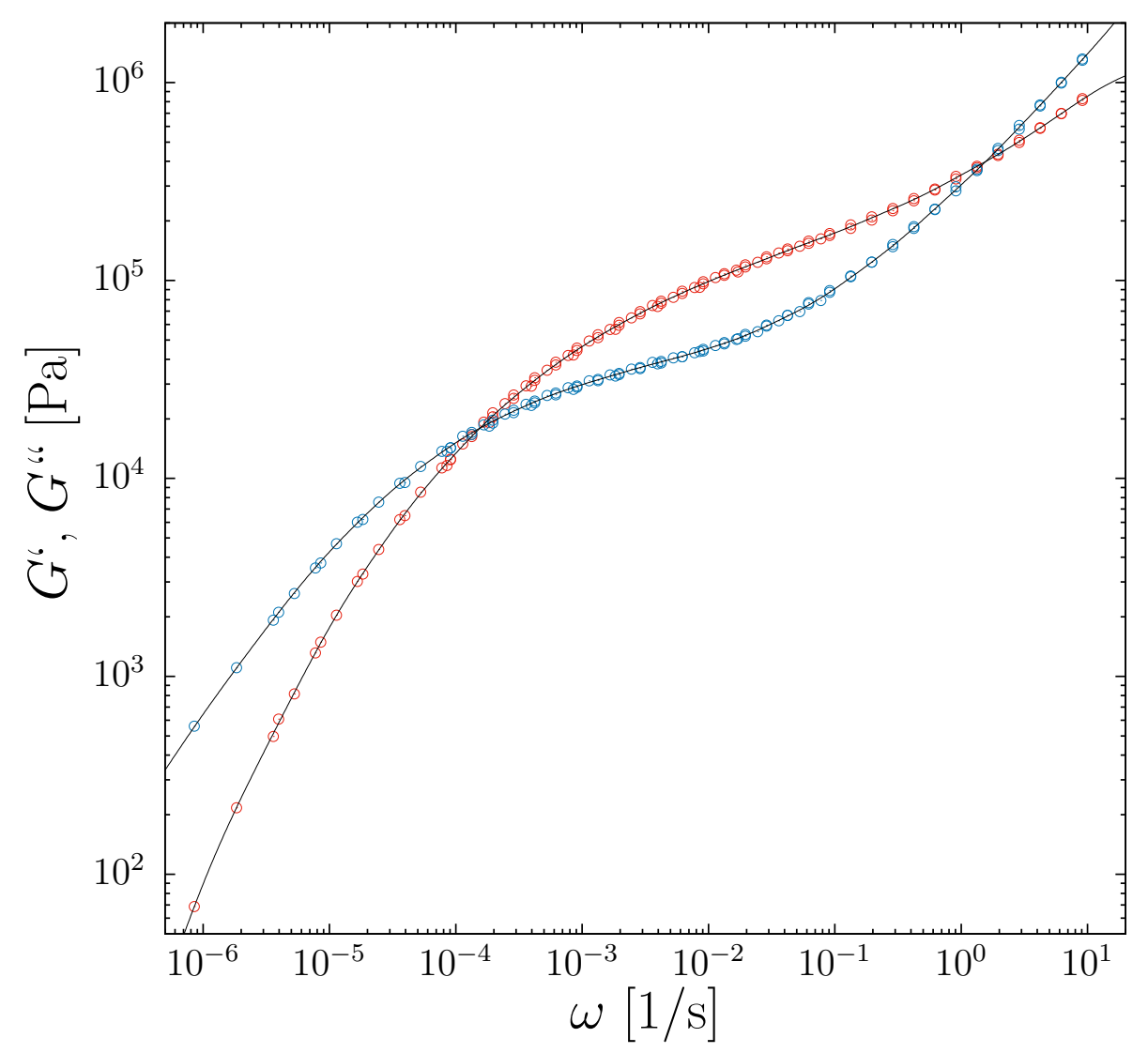

Figure 1: Dynamic mechanical spectroscopical data of polystyrene (CAS 0993-53-6 from Aldrich) measured by [32] in small oscillatory shear flow. The measurements were all time-temperature shifted to $120^{\circ} \mathrm{C}$ here. $G^{\prime}$ (red circle), $G^{\prime \prime}$ (blue circle), and $\omega$ are the storage modulus, the loss modulus and the angular frequency, respectively. The solid lines $(-)$ are the corresponding model predictions from equation (1) with the parameters from the table. 


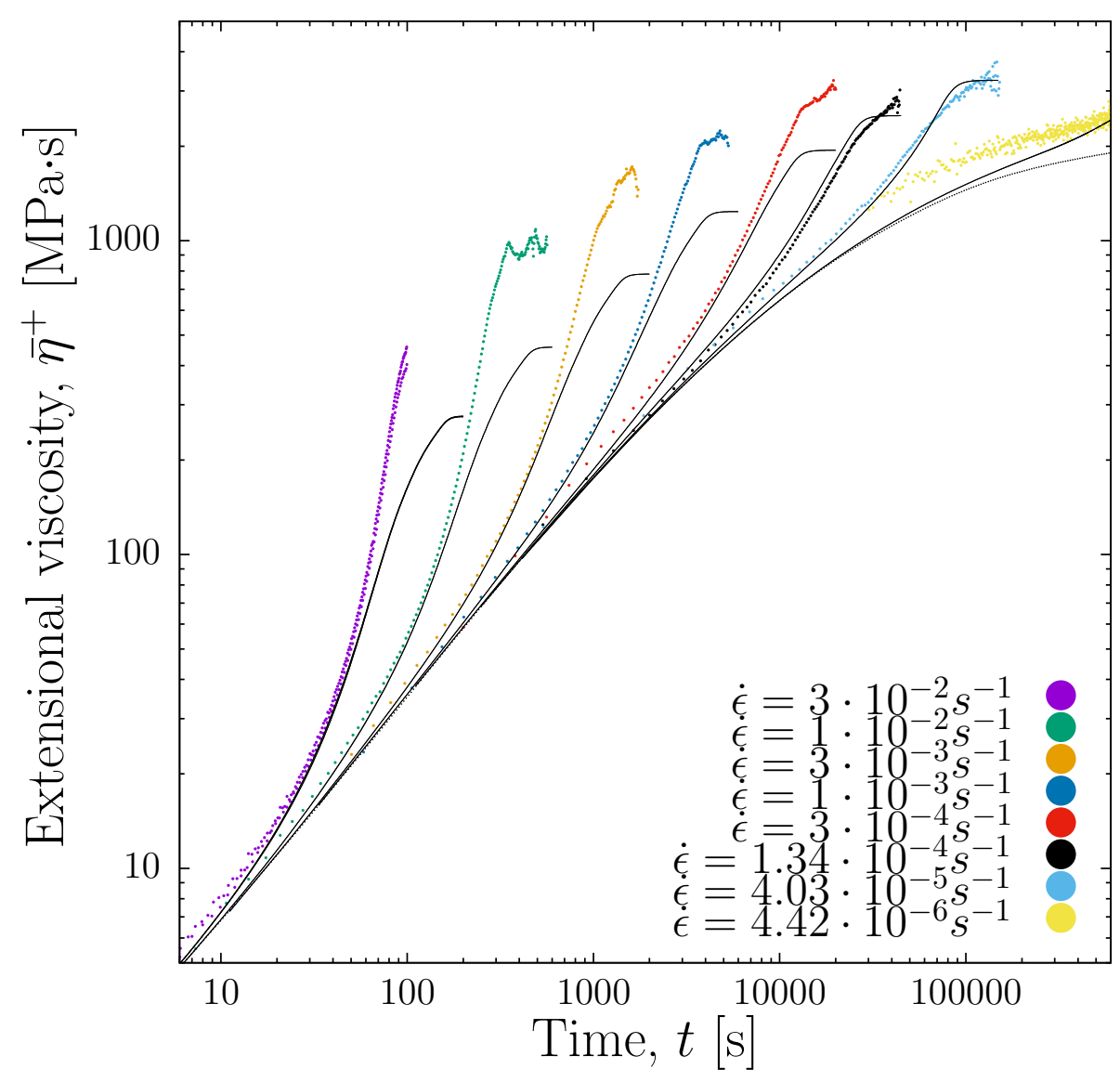

Figure 2: The measured startup viscosity, corrected using equation (2), extended at $120^{\circ} \mathrm{C}$ using extensional rates of $0.03 s^{-1}, 0.01 s^{-1}, 0.003 s^{-1}, 0.001 s^{-1}, 0.0003 s^{-1}, 1.34 \cdot 10^{-4} s^{-1}, 4.03 \cdot 10^{-5} s^{-1}$, and $4.42 \cdot 10^{-6} s^{-1}$. The dotted line $(\cdots)$ is the linear viscoelastic extensional viscosity defined by three times the shear stress growth curve. The solid lines $(-)$ are the model predictions from the equation (4) corresponding to the data using a dilution modulus of $G_{D}=7000 \mathrm{~Pa}$. 


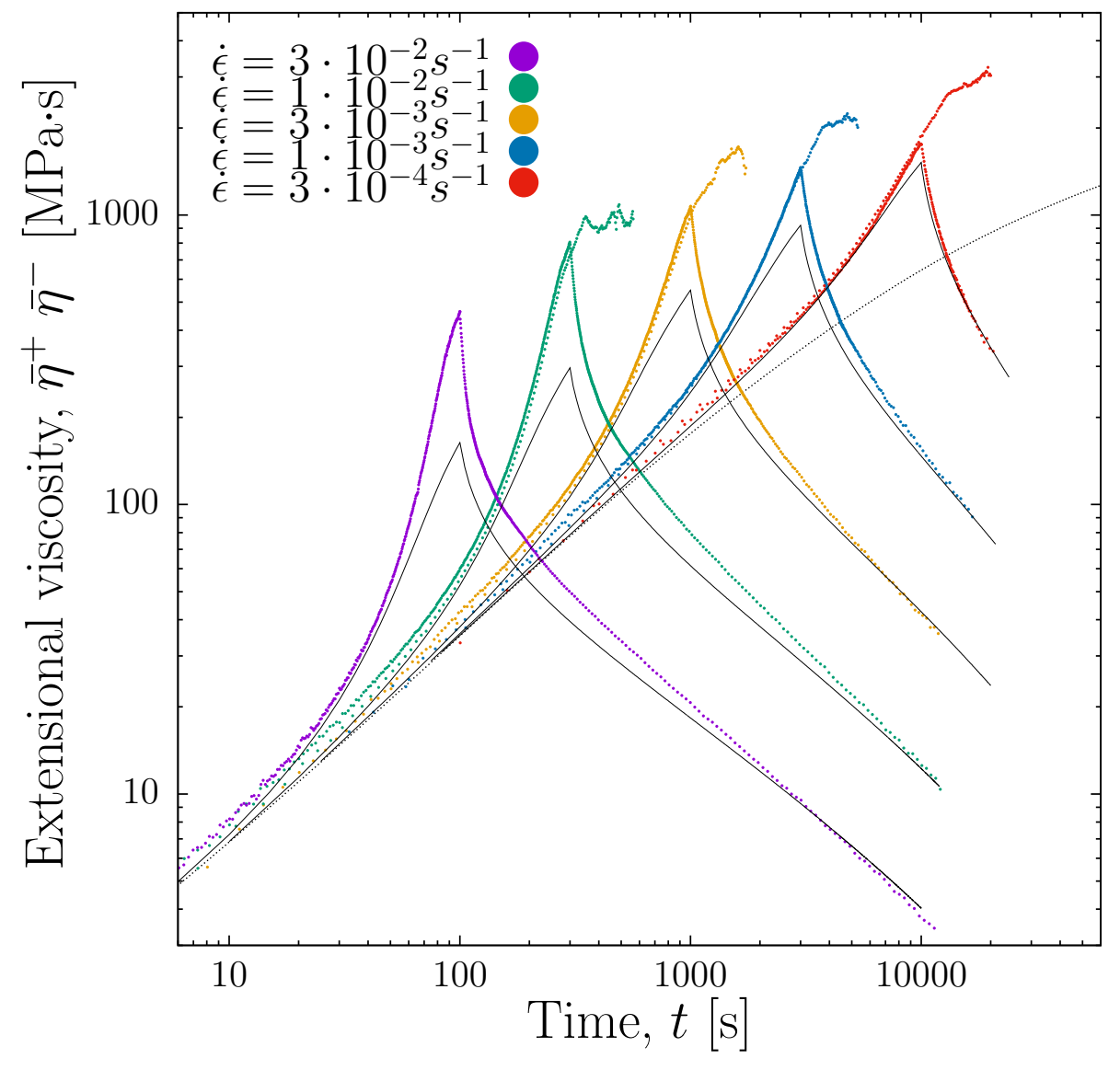

Figure 3: The measured startup and following relaxation viscosity, corrected using equation (2), extended at $120^{\circ} \mathrm{C}$ using extensional rates of $0.03 \mathrm{~s}^{-1}, 0.01 \mathrm{~s}^{-1}, 0.003 \mathrm{~s}^{-1}, 0.001 \mathrm{~s}^{-1}$, and $0.0003 \mathrm{~s}^{-1}$. The relaxations are initiated at a strain on 3 , as the flow is stopped at a strain of 3 and allowed to relax. The dotted line $(\cdots)$ is the linear viscoelastic extensional viscositydefined by three times the shear stress growth curve. Some of the measured startup of uniaxial extensional viscosities, with constant extensional rate, from figure 2 has been added on the figure as well. The solid lines $(-)$ are the model predictions from the equation (4) corresponding to the data using a dilution modulus of $G_{D}=7000 \mathrm{~Pa}$. 


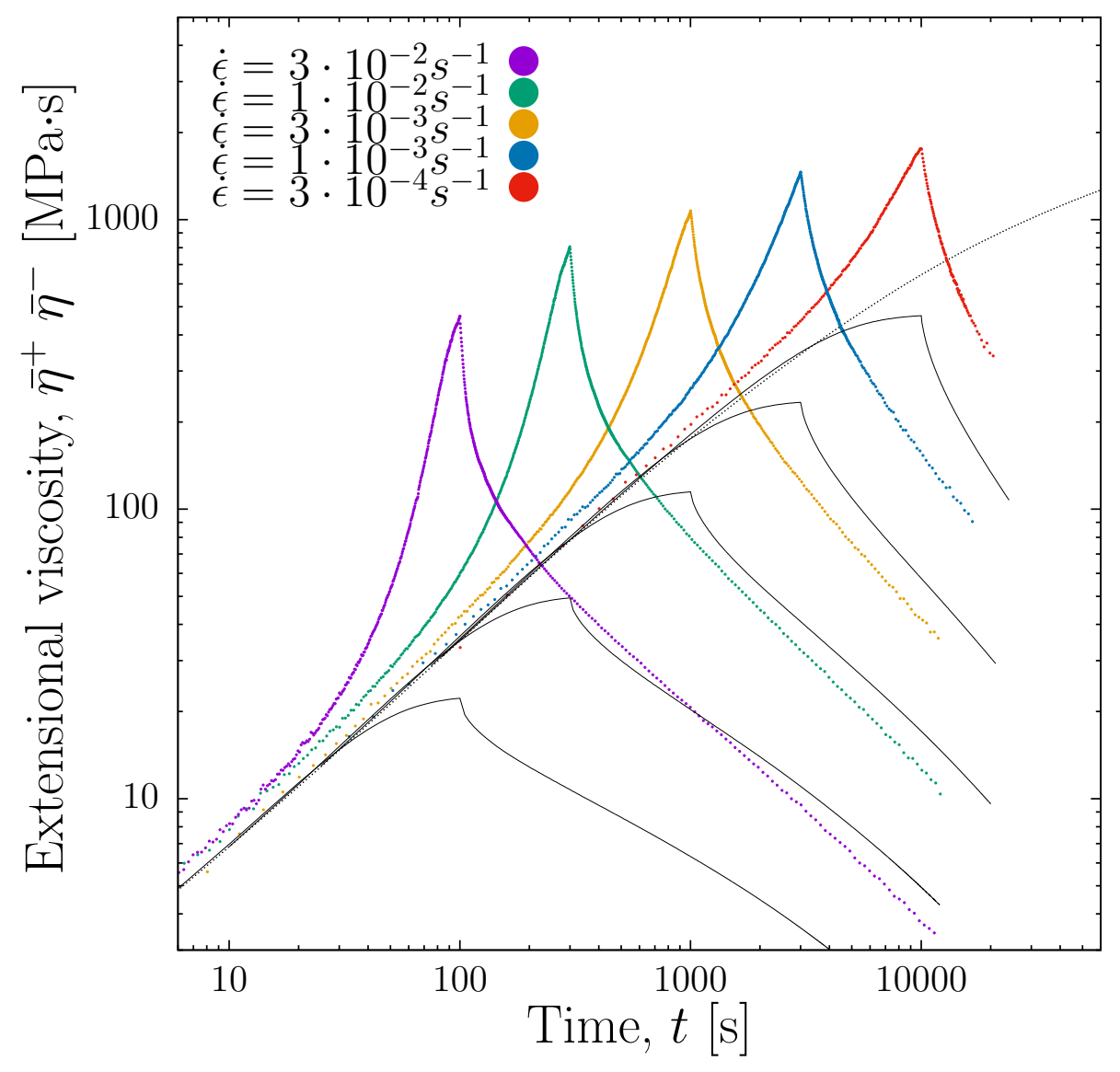

Figure 4: The measured startup and following relaxation viscosity (from figure 3), corrected using equation (2), extended at $120^{\circ} \mathrm{C}$ using extensional rates of $0.03 \mathrm{~s}^{-1}, 0.01 \mathrm{~s}^{-1}, 0.003 \mathrm{~s}^{-1}, 0.001 \mathrm{~s}^{-1}$, and $0.0003 \mathrm{~s}^{-1}$. The relaxations are initiated at a strain on 3 , as the flow is stopped at a strain of 3 and allowed to relax. The dotted line $(\cdots)$ is the linear viscoelastic extensional viscosity defined by three times the shear stress growth curve. The solid lines $(-)$ are the predictions from a pure configurational stress model, corresponding to a lack of internal chain stretch i.e. $f_{i}=1$ in Equation (3). 


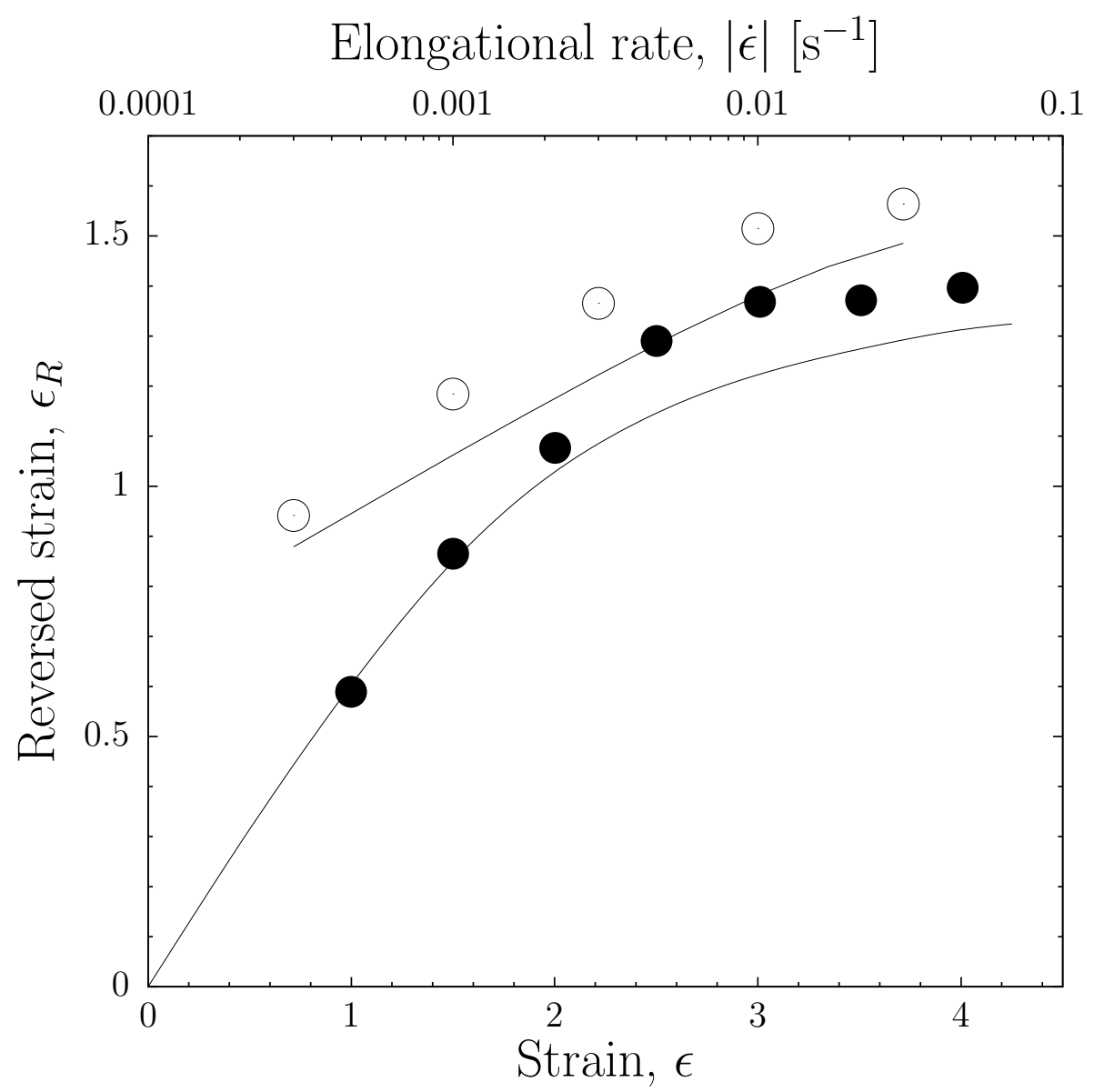

Figure 5: Recovery strain value at $120^{\circ} \mathrm{C}$. The (open) circles referring to the upper abscissa axis showing the elongational rate, where the flow have been reversed at a fixed Hencky strain of 3 . The (solid) bullets, referring to the lower abscissa axis showing Hencky strain where the flow is reversed at a fixed elongational rate of $0.003 s^{-1}$. The lines are the model prediction to the corresponding data using a dilution modulus of $G_{D}=7000 \mathrm{~Pa}$. 


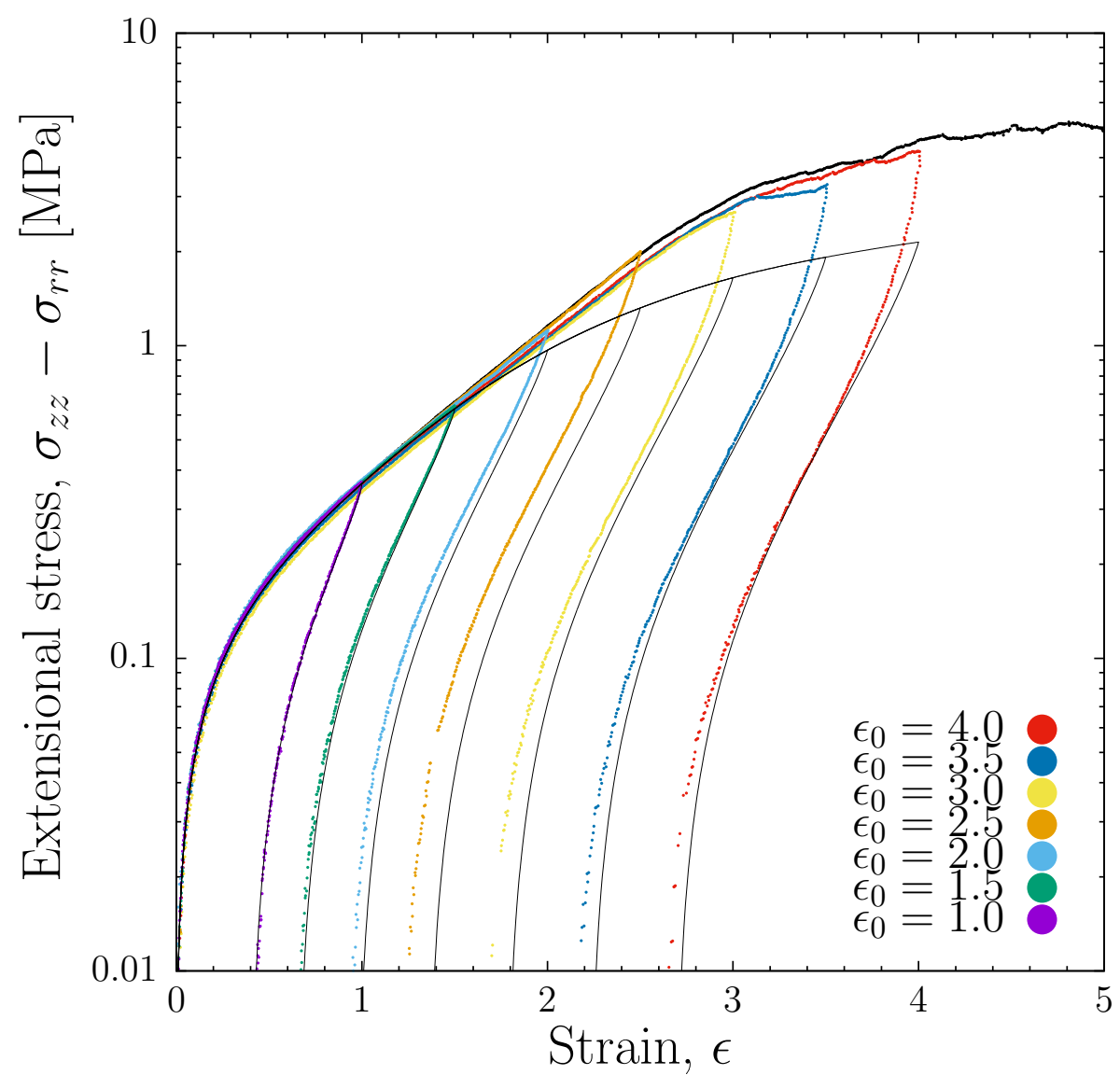

Figure 6: Extension stress, corrected using equation (2), as a function of the strain measured at $120^{\circ} \mathrm{C}$. The flow is startup of extension followed by reversed (biaxial) extension, at a fixed startup of elongational rate of $0.003 \mathrm{~s}^{-1}$. The lines are the model prediction to the corresponding data using a dilution modulus of $G_{D}=7000 \mathrm{~Pa}$. 


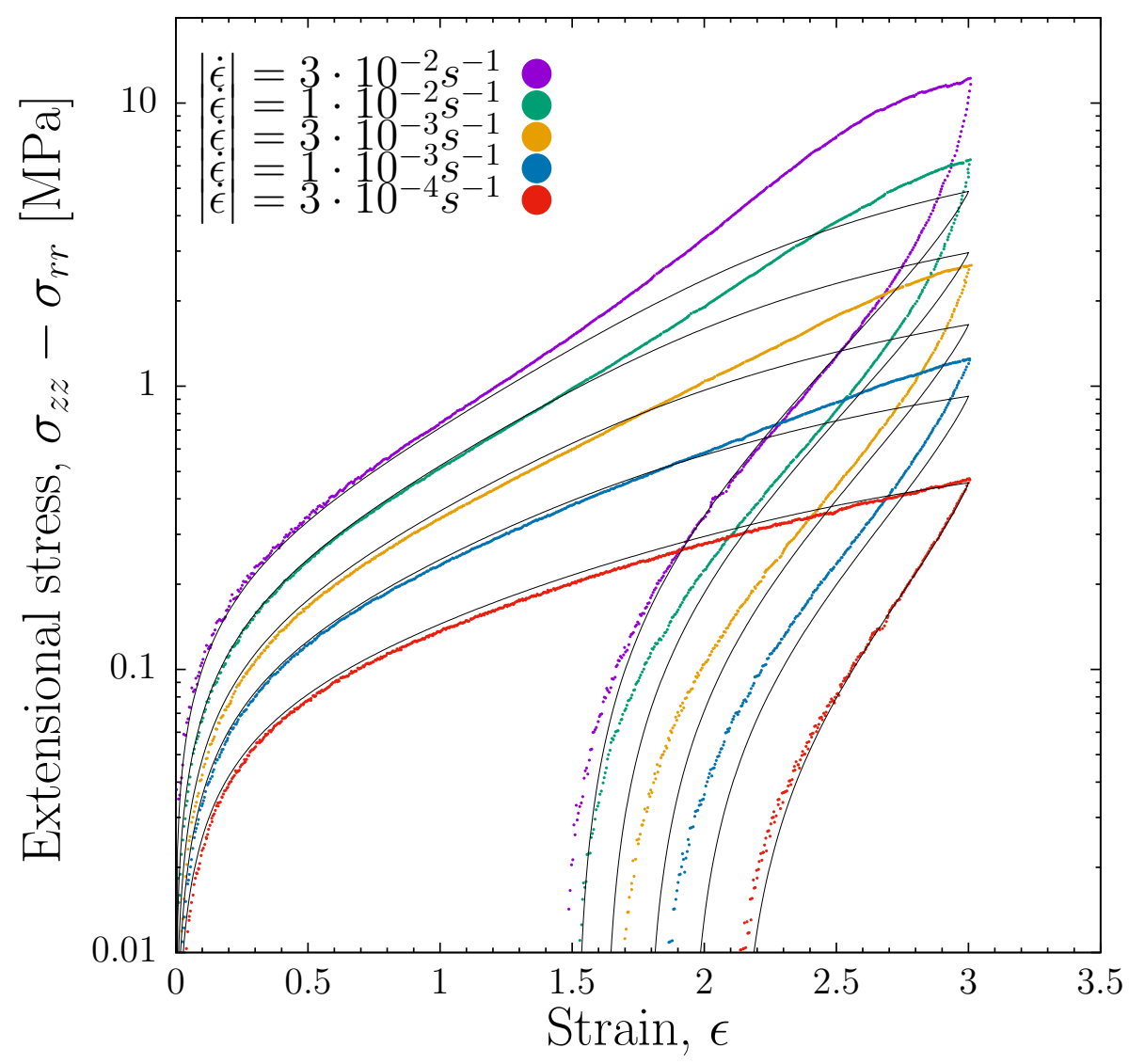

Figure 7: Extension stress, corrected using equation (2), as a function of the strain measured at $120^{\circ} \mathrm{C}$. The flow is startup of extension followed by reversed (biaxial) extension, reversed at a fixed strain of 3 . The lines are the model prediction to the corresponding data using a dilution modulus of $G_{D}=7000 \mathrm{~Pa}$. 


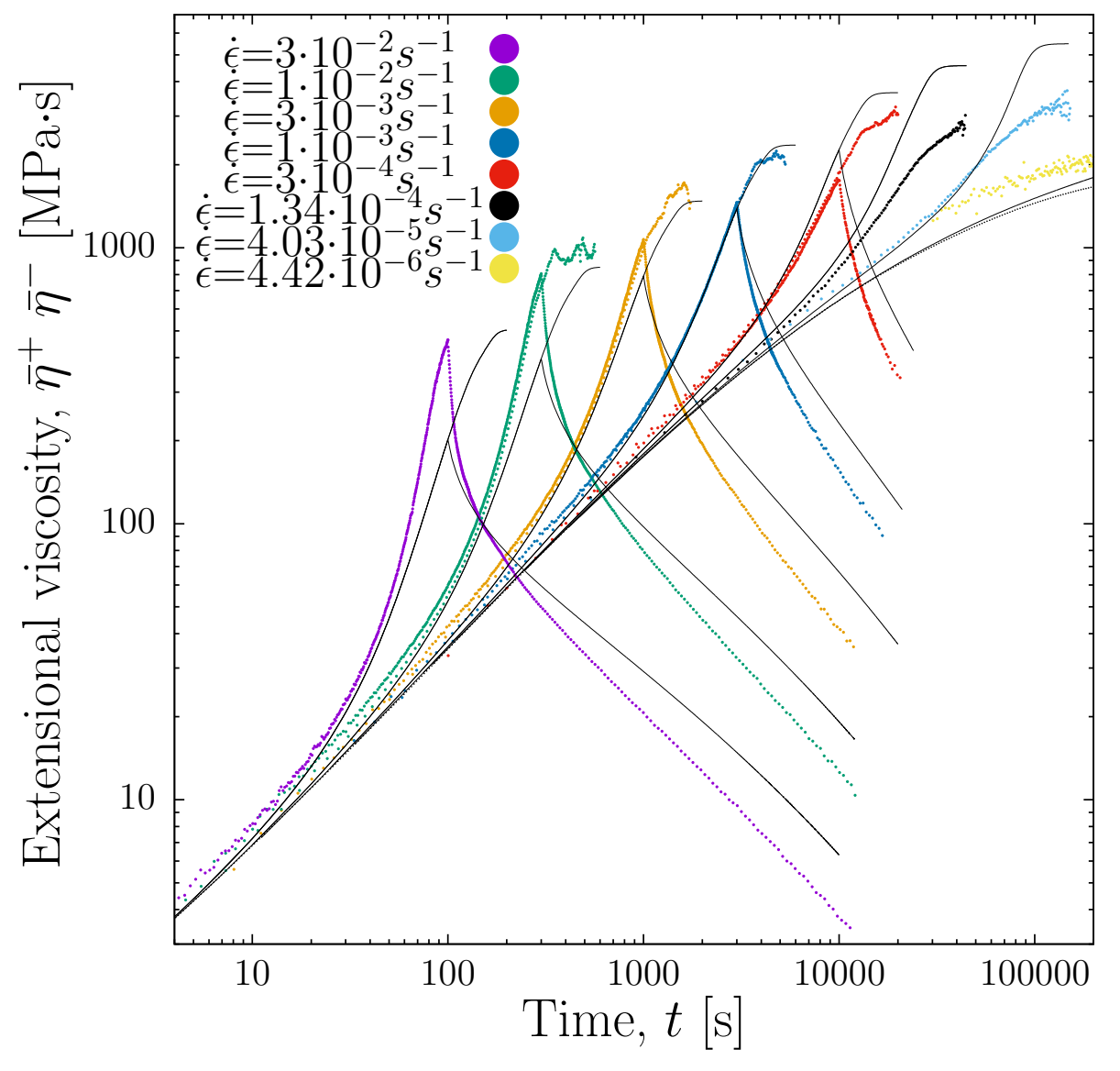

Figure 8: The measured startup and following relaxation viscosity, corrected using equation (2), extended at $120^{\circ} \mathrm{C}$ using extensional rates of $0.03 s^{-1}, 0.01 s^{-1}, 0.003 s^{-1}, 0.001 s^{-1}, 0.0003 s^{-1}$, $1.34 \cdot 10^{-4} \mathrm{~s}^{-1}, 4.03 \cdot 10^{-5} \mathrm{~s}^{-1}$, and $4.42 \cdot 10^{-6} \mathrm{~s}^{-1}$. The relaxations are initiated at a strain on 3 , as the flow is stopped at a strain of 3 and allowed to relax. The dotted line $(\cdots)$ is the linear viscoelastic extensional viscosity defined by three times the shear stress growth curve. Some of the measured startup of uniaxial extensional viscosities, with constant extensional rate, from figure 2 has been added on the figure as well. The solid lines ( - ) are the model predictions from the equation (4) corresponding to the data using a is the dilution modulus $G_{D}=35000 \mathrm{~Pa}$. 